\title{
Analysis of Area Revisitation Patterns in World of Warcarft
}

\author{
Ruck Thawonmas ${ }^{1}$, Keisuke Yoshida $^{1}$, Jing-Kai Lou $^{2}$, and Kuan-Ta Chen ${ }^{2}$ \\ 1 Intelligent Computer Entertainment Lab \\ Graduate School of Science and Engineering, Ritsumeikan University \\ Kusatsu, Shiga, 525-8577, Japan \\ ruck@ci.ritsumei.ac.jp \\ 2 Multimedia Networking and Systems Lab \\ Institute of Information Science, Academia Sinica \\ Taipei 115, Taiwan \\ swc@iis.sinica.edu.tw
}

\begin{abstract}
This paper analyzes area revisitation patterns in World of Warcraft (WoW). Online-game players roam a number of in-game areas while playing the game and revisit some of them with different personal reasons. To clarify this issue, we conduct a large-scale analysis using WoW access log collected for two years consisting of more than sixty thousand characters and have discovered four main groups of area revisitation patterns. We describe also in the paper how our findings can be utilized to support both game developers and players.
\end{abstract}

\section{Introduction}

An online game player regularly revisits some particular areas. Their reasons differ and might be due to several factors, such as player intention, area composition and content. For example, to increase their levels, a group of players might repeatedly visit an area where monsters reside. Another area might be visited routinely because of its periodical game events. Consequently, knowledge on area groups, each with similar revisitation characteristic, has high potential in supporting of not only game developers but also players.

In this paper, inspired by a recent work on analysis of web revisitation patterns [1], we hypothesize that, similar to web pages, typical patterns exist in revisitation of online-game areas. To validate our hypothesis, we conduct a large-scale analysis using access log from World of Warcraft (WoW), a Massively Multiplayer Online Role-Playing Game (MMORPG). The contributions of this work are (a) the first analysis of this kind on an online game, (b) four area-revisitation patterns (or groups) found from access log of the world most popular online game WoW, (c) thorough discussions on each pattern, and (d) our implications to applications of the findings. 


\section{Data Acquisition and Filtering}

\subsection{Data Acquisition}

In this work, we use access log from a Taiwanese server of WoW. At present, WoW has the highest number of subscribers 2 . WoW players must choose to belong to either Alliance or Horde faction from the beginning. Access log of Horde characters was acquired by executing / who, at our client every ten minutes during two years from January 2006 to December 2007. Before filtering, our log contains more than sixty thousand characters of all Horde races: Blood Elf, Orc, Tauren, Troll, and Undead. An execution of / who gives us information on all currently-logging-in characters and their playing areas at the execution time; other information includes the race, job, and level of the corresponding character.

\subsection{Data Filtering}

To increase the reliability in the data, we removed characters with total play time below 40 hours, an arguable standard RPG length. We further removed characters with at least one of the four attributes falling below 10th percentile against other characters. These four attributes are the total numbers of (a) area transitions, (b) unique areas visited, (c) and areas revisited, as well as (d) the area-transition ratio. The last one is the ratio between (a) and the log length of the character. Table 1 shows an example access log, a simplified one though, of a character. For this character, the aforementioned attributes are $6,4,3$, and 0.6 , respectively. Note that because this character consecutively visited area $\mathrm{D}$, its total number of areas revisited is 3, not 4, i.e., revisiting Area A in 22 hours and 40 minutes, Area B in 50 minutes, and Area C in 22 hours and 10 minutes.

To exclude outliers, such as bots [3] and extremely hardcore players who spend an extraordinary amount of time in the game, we further filtered out characters

Table 1. Example of simplified access log for explanation of four character attrbutes

\begin{tabular}{|c|c|}
\hline Time & Area \\
\hline 10/08/06 $02: 12: 37$ & $\overline{\mathrm{A}}$ \\
\hline 10/08/06 02:22:28 & $\mathrm{A}$ \\
\hline 10/08/06 $02: 32: 37$ & B \\
\hline 10/08/06 $02: 42: 38$ & $\mathrm{C}$ \\
\hline 10/08/06 03:02:30 & $\mathrm{D}$ \\
\hline 10/08/06 $03: 12: 37$ & $\overline{\mathrm{D}}$ \\
\hline 10/08/06 03:22:35 & $\mathrm{B}$ \\
\hline 10/09/06 $00: 52: 37$ & $\mathrm{C}$ \\
\hline 10/09/06 01:02:37 & $\mathrm{A}$ \\
\hline $10 / 12 / 06 \quad 23: 36: 37$ & $\mathrm{~A}$ \\
\hline
\end{tabular}


with log length of 99th percentile against other characters. As a result, the number of remaining characters becomes 6491. For area filtering, we removed areas with the integral of the revisitation curve, defined in the next section, of 1st percentile against other areas. This results in 107 areas remaining after filtering.

\section{Analysis}

Our analysis approach follows the recipe in [1]. First, we use the revisitation curve to characterize an area of interest. Then we cluster these areas into related groups using a hierarchical clustering algorithm.

The revisitation curve of an area represents the total number of times the area is revisited by characters within in a given time interval. Thirteen exponential time bins are employed, i.e., within 32, 64, 98, 136, 212, 424, 848, 1696, 3392, 6784, 13568, $27136 \mathrm{mins}$, and above; representing approximately, $30 \mathrm{~min}, 1$ hour, 1.5 hour, 2 hours, 3 hours, 6 hours, 12 hours, 1 day, 2 days, 4 days, 8 days, 16 days, and above, respectively. For the example log in Table 11 the data of this character will add 1 to the 2nd bin of Area B and to the 8th bin of Areas A as well as C. To facilitate comparisons between areas, normalization is performed for the revisitation curve of area $[i]$ as follows:

$$
\frac{\operatorname{bin}[j] \text { of area }[i]}{\text { average of } \operatorname{bin}[j] \text { among all areas }}
$$

For area $[i]$, each of the thirteen normalized bin values represents an element of 13-dimensional input vector $i$ used in cluster analysis described below.

To cluster areas into multiple groups, each having similar revisitation characteristic, we adopt commonly used Ward hierarchical clustering 4, available in R. Because shape information is important, the cosine distance is selected in Ward clustering. The index in use for automatically deciding the number of clusters is maximization of

$\frac{\text { interclass variance/(number of clusters }-1)}{\text { intraclass variance/(number of areas - number of clusters })}$

[5]. Because we anticipate that there should also be four main groups as in [1], the minimum number of clusters is set to four.

\section{Results and Discussions}

Figure 1 Shows the clustering results where all areas are divided into 15 clusters. Each cluster is labeled to one of the four groups, i.e., Fast, Medium, Slow, and Hybrid, based on the revisitation curve's shape of the cluster centroid. Table 2 summarizes the cluster information for Fast, Medium, and Slow; and Table 3 


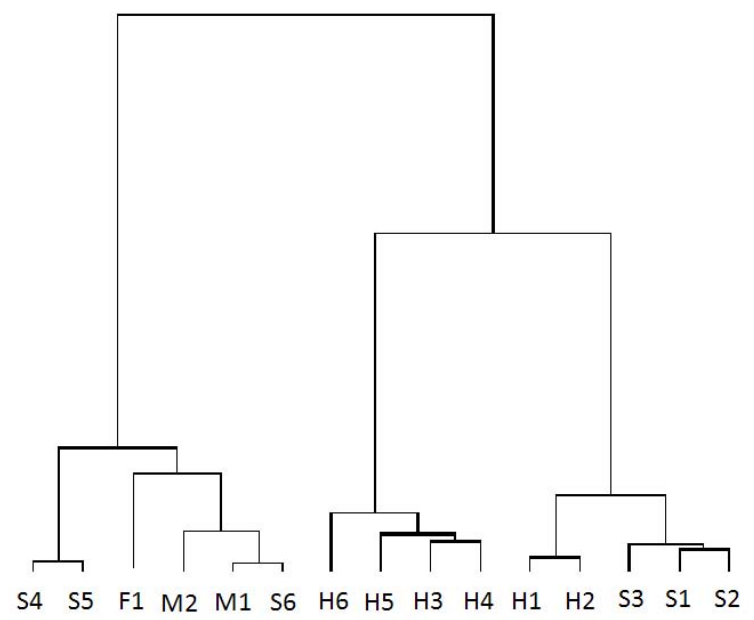

Fig. 1. Hierarchical clustering results

summarizes the cluster information for Hybrid. Each group is discussed in detail in the following.

\section{$4.1 \quad$ Fast}

Areas in this group are often revisited within a short interval, say, less than hour. The areas in this group can be classified into two categories: battlegrounds and starting areas.

\subsubsection{Battleground}

One of the main features of the Warcraft universe is the raging conflict between Horde and the Alliance. Battlegrounds are instanced areas solely created for PvP (player-versus-player) combats. The two initial Battleground areas, Alterac Valley and Warsong Gulch, went online on June 7th, 2005. Arathi Basin was introduced in Patch 1.7 on September 13th, 2005. The fourth battleground, named Eye of the Storm, was released with the Burning Crusade extension pack.

Battlegrounds are instances and have a limit on the number of players who can enter at the same time, where the limit varies in different battlegrounds. The game provides Battleground Queuing System to assist players register for the join battlegrounds given the player number limits. Players can wait for entering a battleground anywhere in the world via the game's user interface. Once it is the turn for a player to enter the battleground, he will be teleported into the battleground, and teleported back after the battle finishes. All the battlegrounds are classified as fast-revisiting areas, which indicates that a battle is usually finished in 30 minutes and PvP players often reenter a battleground immediately for the next match. 
Table 2. Summary of cluster information for Fast, Medium, and Slow

\begin{tabular}{|c|c|c|c|c|}
\hline Group & Cluster & Centroid Curve & $\begin{array}{c}\text { Number } \\
\text { of } \\
\text { Areas }\end{array}$ & Area Names \\
\hline Fast & F1 & & 7 & $\begin{array}{c}\text { Alterac Valley, Arathi Basin, } \\
\text { Eversong Woods, Eye of the Storm, } \\
\text { Ghostlands, Maraudon, } \\
\text { Warsong Gulch }\end{array}$ \\
\hline \multirow[t]{2}{*}{ Medium } & M1 & & 8 & $\begin{array}{c}\text { Blade's Edge Mountains, } \\
\text { Hellfire Peninsula, Nagrand, } \\
\text { Netherstorm, Shadowmoon Valley, } \\
\text { Terokkar Forest, Undercity, } \\
\text { Zangarmarsh } \\
\end{array}$ \\
\hline & $\mathrm{M} 2$ & & 2 & Orgrimmar, Shattrath City \\
\hline \multirow{6}{*}{ Slow } & S1 & & 6 & $\begin{array}{l}\text { Duskwood, Elwynn Forest, } \\
\text { Loch Modan, Redridge Mountains, } \\
\text { Twisting Nether, Wetlands }\end{array}$ \\
\hline & S2 & & 5 & $\begin{array}{l}\text { Blackfathom Deeps, } \\
\text { Ragefire Chasm, Razorfen Downs, } \\
\text { Razorfen Kraul, Shadowfang Keep }\end{array}$ \\
\hline & S3 & & 11 & $\begin{array}{c}\text { Blasted Lands, Burning Steppes, } \\
\text { Darkshore, Dustwallow Marsh, } \\
\text { Hall of Legends, Moonglade, } \\
\text { Mulgore, Scarlet Monastery, } \\
\text { Swamp of Sorrows, } \\
\text { The Temple of Atal'Hakkar, } \\
\text { Wailing Caverns }\end{array}$ \\
\hline & S4 & & 14 & $\begin{array}{c}\text { Alterac Mountains, } \\
\text { Arathi Highlands, Ashenvale, } \\
\text { Azshara, Badlands, Desolace, } \\
\text { Feralas, Hillsbrad Foothills, } \\
\text { Silverpine Forest, Tanaris, } \\
\text { The Hinterlands, } \\
\text { Thousand Needles, Thunder Bluff, } \\
\text { Un'Goro Crater }\end{array}$ \\
\hline & S5 & & 6 & $\begin{array}{c}\text { Blackrock Mountain, } \\
\text { Deadwind Pass, Durotar, } \\
\text { Gates of Ahn'Qiraj, Searing Gorge, } \\
\text { Tirisfal Glades }\end{array}$ \\
\hline & S6 & & 9 & $\begin{array}{l}\text { Eastern Plaguelands, Felwood, } \\
\text { Silithus, Silvermoon City, } \\
\text { Stonetalon Mountains, } \\
\text { Stranglethorn Vale, The Barrens, } \\
\text { Western Plaguelands, Winterspring }\end{array}$ \\
\hline
\end{tabular}

\subsubsection{Starting Area}

During our data collection period (Jan 2006 to Dec 2007), an extension pack called "The Burning Crusade" was released in March 2007. In this extension pack, there are two new races introduced: The Draenei for the Alliance and the Blood Elves for the Horde. New Blood Elf characters start their journey in the Eversong Woods, the northern section of Quel'Thalas. Farther south is the Ghostlands, a level 10-20 zone. Low-level Blood Elves gain experience points and reward items by pursuing the quests in Eversong Woods and Ghostlands. However, most of the quest givers are in the capital city, Silvermoon (S6), which 
Table 3. Summary of cluster information for Hybrid

\begin{tabular}{|c|c|c|c|c|}
\hline Group & Cluster & Centroid Curve & $\begin{array}{c}\text { Number } \\
\text { of } \\
\text { Areas }\end{array}$ & Area Names \\
\hline \multirow{6}{*}{ Hybrid } & H1 & & 11 & $\begin{array}{c}\text { Dire Maul, Hellfire Ramparts, } \\
\text { Scholomance, Shadow Labyrinth, } \\
\text { Stratholme, The Arcatraz, } \\
\text { The Black Morass, The Botanica, } \\
\text { The Shattered Halls, } \\
\text { The Steamvault, The Underbog }\end{array}$ \\
\hline & $\mathrm{H} 2$ & $v$ & 10 & $\begin{array}{c}\text { Auchenai Crypts, } \\
\text { Blackrock Depths, Gnomeregan, } \\
\text { Mana-Tombs, } \\
\text { Old Hillsbrad Foothills, } \\
\text { Sethekk Halls, The Blood Furnace, } \\
\text { The Slave Pens, Uldaman, } \\
\text { Zul'Farrak }\end{array}$ \\
\hline & $\mathrm{H} 3$ & & 5 & $\begin{array}{c}\text { Blackrock Spire, Naxxramas, } \\
\text { Ruins of Ahn'Qiraj, The Mechanar, } \\
\text { Zul'Gurub }\end{array}$ \\
\hline & $\mathrm{H} 4$ & & 3 & $\begin{array}{c}\text { Blade's Edge Arena, } \\
\text { Nagrand Arena, Ruins of Lordaeron }\end{array}$ \\
\hline & H5 & & 5 & $\begin{array}{l}\text { Gruul's Lair, Magtheridon's Lair, } \\
\text { Molten Core, Onyxia's Lair, } \\
\text { Tempest Keep }\end{array}$ \\
\hline & H6 & 々 & 5 & $\begin{array}{c}\text { Ahn'Qiraj, Blackwing Lair, } \\
\text { Karazhan, Serpentshrine Cavern, } \\
\text { Zul'Aman }\end{array}$ \\
\hline
\end{tabular}

is located in the northeastern part of the Eversong Woods. Therefore, low-level Blood Elves have to regularly return to Silvermoon to complete their quests or to take new quests. After that, they tend to go back to the starting areas and carry on their new quests. The fast revisitation of starting areas confirms that the quests for low-level players are relative easy and can be done within a short time.

\subsection{Medium}

Areas in this cluster have a peak in the left middle of the curve, i.e., between 1 hour and 1 day. We find that the areas in this cluster are due to two possible reasons as follows.

\subsubsection{Daily Quest}

Daily quests (often called "dailies") are repeatable quests firstly introduced in Patch 2.1. Areas for daily quests are included in M1, such as etherstorm and Shadowmoon Valley. These quests provide significantly better rewards than regular quests. A player can complete up to 25 daily quests in each day. After a player solves 25 daily requests, he must wait for the "daily quest reset" event. The exact time for such reset depends on the configuration of each realm, which is often chosen in the early morning. Thus, it is reasonable for players to revisit the areas which provide daily quests between 1 hour and 1 day. 


\subsubsection{Bank and Auction House}

Other areas in the Medium cluster are capital cities, including Undercity (M1) for the Undead, Orgrimmar (M2) for the Orc and Troll, and Shattrath city (M2) for the united force. The cities serve as the main cultural, political, and economical center of each race. To players, one of the main functionalities of the capital cities is their economical functions, i.e., banking and auction. Banks and auction houses are usually located only in major cities or towns, especially in capital cities. A bank is a building with teller NPCs which allow a player to access his own private storage. A player can freely deposit and withdraw his items and currency in the storage via a bank or its branches. An auction house is a place for players to trade goods, weapons, and equipments. As of the release time of Patch 1.9, each capital city has its own auction house. The medium revisitation patterns of capital cities implies that players tend to regularly come back to their respective capital cities to access their private storage or trade goods with others.

\subsection{Slow}

The revisitation curves in this cluster have a slow peak, i.e., revisitation intervals are longer than 1 day. The level 30-70 areas are included. The revisitation curves of growth areas for moderate and upper level (30-70) characters, included in S4, S5, S6, have higher amplitudes than those of lower-level growth areas.

\subsubsection{Low-Level Instance}

Those instances are special areas where a group of players can interact privately within a dungeon. Instance dungeons tend to feature the most difficult and rewarding content. Since the majority of the players had achieved the 70th level in our traces, the low-level (20-30 level) instances, such as Blackfathom Deeps, Ragefire Chasm, Razorfen Downs, Razorfen Kraul, and Shadowfang Keep (all in S2), can no longer offer satisfying rewards. Such low-level instances thus become less popular, and therefore, players do not revisit the areas frequently.

\subsubsection{Monthly Event}

Another reason for slow revisitations is due to a monthly event, the Darkmoon Faire (the Faire), which is a regular event firstly introduced in Patch 1.6. The Faires always start on the first Monday of a month, and the location rotates among three places, namely, Elwynn Forest (S1), Mulgore (S3), and Terokkar Forest (M1). The event lasts for one week and appears in turn in the three places each month. Because some special goods can only be purchased in the Fairs, players may manage to attend such events, even the location is far away from their homelands. For example, when the Faire takes place in the Elwynn Forest near the Alliance land, we find that some Horde players traveled through Dust Wood, Loch Modan, Redridge Mountains, Twisting Nether, and Wetland (all in S1) in order to get to Elwynn Forest. Because these in-between areas are unpopular and may be only visited due to such rare events, those areas exhibit slow revisitation patterns and are included in the slow revisitation cluster. 


\subsection{Hybrid}

\subsubsection{High-Level Instance}

High-level instances feature the most difficult and rewarding content, thus such instances are very popular. For example, Stratholme, Old Hillsbrad Foothills, Blackrock Spire, Molten Core, and Ahn'Qiraj which associate with H1, H2, H3, H5, and $\mathrm{H} 6$, respectively, are in this category. Due to the challenge in solving quests offered in these areas, very often the entire party of players are eliminated by the enemies and transferred to the nearest land outside the instance. This kind of situation is called the "wipeout". After a wipeout, players usually gather and enter the same instance within 10 minutes, which causes the left peak in the revisitation curve.

On the other hand, in order to control the number of valuable items from inflating, an instance may have a "lockout" period after it is conquered. During the lockout period, the players who completed the instance are not allowed to re-enter the instance. The lockout period is often set to one day or one week depending on the scarcity of the reward. This design leads to the right peak in the revisitation curves for these areas.

\subsubsection{Arena}

The remaining areas in the Hybrid cluster are mostly arenas, including Blade's Edege Arena, Nagrand Arena, and Ruins of Lordaeron (all in H4). An arena is a place in which teams of players compete against each other to gain the Arena Points in deathmatch-like player-vs-player combats. Since such matches often conclude in 10 minutes, players tend to reenter an arena frequently. This is the reason for the left peak.

However, the Arena Points are not awarded after each match. For example, one requirement to gain the Arena Points is to attend at least ten matches within a week, and the Points is awarded on Thursdays regularly. Therefore, players tend to reenter an arena to gain rewarding points weekly. We consider this design the cause to the right peak in the revisitation curve.

\section{$5 \quad$ Design Implications}

Design implications are given in this section. Although WoW is used in the analysis in this paper, we note here that our implications are applicable to other MMORPGs.

\subsection{Area Design Reconsideration}

Through the analysis of players' area revisitation patterns, game designers can check whether the usage of areas conforms to their expectation. Specifically, some areas may be unreasonably popular where players revisit them very frequently, while some others may be unexpectedly unpopular where players seldom revisit. The unexpectedly popular areas might be because players have found some tricks that can defeat "boss" monsters or gain a great quantity of reward, whereas the unexpectedly unpopular areas might be because the game missions provided are 
too difficult or the reward is not worth enough. Thus, we consider area revisitation patterns could be a useful summarization tool of game players' passion about certain areas, and, at the same time, a useful analytical tool for game designers to reconsider the design of game areas.

In addition, to maintain high user satisfaction, the contents of Fast and Medium areas should be updated with higher frequencies than those of Slow. Fast and Medium areas are more popular ones where players more frequently visit. Neglect in updating them may lead to player retirement.

\subsection{Players' Social Network Solidification}

For a group of players, if their characters share similar area revisitation patterns, it is likely that they share similar game play preference and strategies, and their characters have same game levels and expertise. For example, a group of players may all prefer to boost the game levels through slashing monsters in caves rather than simply chatting with other players in towns. On the other hand, some other players may prefer accumulating valuables by manufacturing goods, provide services, or trading, thus they tend to spend a lot of time in market places and rarely get into battlefield areas. Via the clustering of area revisitation patterns we are able to perform player clustering, where players in the same cluster share similar game play preference and interests.

Game operators can exploit this kind of information to solidify the social network between gamers by designing systems like "friend recommendation" or by holding social events that encourage players to know about each other. For players who share similar game play strategies, they are more likely to connect to and share with each other, which will establish social relationships between those players and further solidify the overall social network of the game's participants. Consequently, this will increase the stickiness to the game and furthermore prolong the game's lifetime.

\subsection{Player Support}

Individual player support can be provided based on area revisitation characteristic of a player of interest. For example, assume that the revisitation curves of a character of interest to battle areas exhibit Slow, but those to growth areas are Fast. This indicates that the player does not favor fighting against other players, but rather prefers to raise his/her character's level. The game system can thus give to this player personal supports such as a direct invitation to an event at a growth area. In addition, the game system can provide to players a kind of reminder service that reminds a player of interest to visit an area whose blank interval since the last visit has passed the peak time in the revistation curve.

If an area search function is available in the game, information on area revisitation can be used in area retrieval computation. For example, assigning more preferences to Slow areas, than to Fast or Medium areas, in the search result might give players higher satisfaction. This is because we think there is a higher possibility that players use the area search function to locate less known areas, such as those in Slow group. 


\section{Related Work}

Since an early report [6] on a large amount of re-visit information in web navigation, many studies [7-11] have been conducted to understand webpage revisitation and browsing behavior. Compared to these studies, the work in [1] that we base on is the largest study in terms of the number of participants. Very recently, the same group of authors has furthered their work and summarized their recent findings on the association between change in content and revisitation in [12.

WoW has been used as a research platform in many studies because of its popularity and availability of WoW API [13, allowing interaction and modification of the game. In 14] and [15, automatically collected data were explored to understand gaming experiences and social dynamics, respectively. The predictability of online-game players' subscription time and the effect of changes in game features to social interaction were studied in [16] and [17, respectively. Very recently, a system for automatically generating comics from WoW gaming experiences has been developed [18.

\section{Conclusions}

Inspired by an existing work on analysis of web revisitation patterns, in this paper, we analyzed area revisitation patterns in WoW. As in web, areas were divided into four groups Fast, Medium, Slow and Hybrid according to their revisitation curves. For each group, we highlighted representative areas and their characteristics associated with their curves. A number of design implications were given that support not only the game-developer side but also the game-player side. Extensive tests of these implications are left as our future work.

\section{Acknowledgments}

This work was supported in part by Japan Society for Promotion of Science (JSPS) under the Grant-in-Aid for Scientific Research (C) 20500146 as well as by the National Science Council under the grant NSC97-2221-E-001-009 (Taiwan).

\section{References}

1. Adar, E., Teevan, J., Dumais, S.: Large Scale Analysis of Web Revisitation Patterns. In: Proc. of the twenty-sixth annual SIGCHI conference on Human factors in computing systems (CHI 2008), pp. 1197-1206 (2008)

2. http://www.mmogchart.com/Chart1.html

3. Thawonmas, R., Kashifuji, Y., Chen, K.T.: Detection of MMORPG Bots Based on Behavior Analysis. In: Proc. of ACM SIGCHI International Conference on Advances in Entertainment Technology (ACE 2008), pp. 91-94 (2008)

4. Ward, J.H.: Hierarchical Grouping to optimize an objective function. Journal of American Statistical Association 58(301), 236-244 (1963) 
5. Calinski, T., Harabasz, J.: A dendrite method for cluster analysis. Communications in statistics 3(1), 1-27 (1974)

6. Catledge, L.D., Pitkow, J.E.: Characterizing browsing strategies in the WorldWide Web. In: Proc. of the Third International World-Wide Web conference on Technology, tools and applications (WWW 1995), pp. 1065-1073 (1995)

7. Tauscher, L., Greenberg, S.: How people revisit Web pages: Empirical findings and implications for the design of history systems. Int. J. of Human-Computer Studies 47(1), 97-137 (1997)

8. Cockburn, A., McKenzie, B.: What do Web users do? An empirical analysis of Web use. Int. J. of Human-Computer Studies 54(6), 903-922 (2001)

9. Herder, E.: Characterizations of user Web revisit behavior. In: Proc. of Workshop on Adaptivity and User Modeling in Interactive Systems (ABIS 2005), pp. 32-37 (2005)

10. Kellar, M., Watters, C., Shepherd, M.: A goal-based classification of Web information tasks. In: Proceedings of the Annual Meeting of the American Society for Information Science and Technology (ASIS\&T 2006) (CD-ROM Proceedings) (2006)

11. Obendorf, H., Weinreich, H., Herder, E., Mayer, M.: Web page revisitation revisited: Implications of a long-term click-stream study of a Long-term Click-stream Study of Browser Usage. In: Proc. of the twenty-fifth annual SIGCHI conference on Human factors in computing systems (CHI 2007), pp. 597-606 (2007)

12. Adar, E., Teevan, J., Dumais, S.: Resonance on the Web: Web Dynamics and Revisitation Patterns. In: Proc. of the twenty-seventh annual SIGCHI conference on Human factors in computing systems (CHI 2009), pp. 1381-1390 (2009)

13. http://www.wowwiki.com/World_of_Warcraft_API

14. Ducheneaut, N., Yee, N., Nickell, E., Moore, R.J.: Building a MMO with mass appeal: a look at gameplay in World of Warcraft. Games and Culture 1(4), 281317 (2006)

15. Williams, D., Ducheneaut, N., Xiong, L., Zhang, Y., Yee, N., Nickell, E.: From tree house to barracks: The social life of guilds in World of Warcraft. Games and Culture 1(4), 338-361 (2006)

16. Tarng, P.-Y., Chen, K.T., Huang, P.: An Analysis of WoW Players' Game Hours. In: Proc. of the seventh annual Workshop on Network and Systems Support for Games, NetGames 2008 (2008)

17. Chen, V., Duh, H., Hong, R.: The Changing Dynamic of Social Interaction in World of Warcraft: The Impacts of Game Feature Change. In: Proc. of ACM SIGCHI International Conference on Advances in Entertainment Technology (ACE 2008), pp. 356-359 (2008)

18. Chan, C.J., Thawonmas, R., Chen, K.T.: Automatic Storytelling in Comics: A Case Study on World of Warcraft. In: CHI Extended Abstracts 2009, pp. 35893594 (2009) 